\title{
SELECCIÓN DE PROVEEDORES BAJO UNA ESTRATEGIA DE ABASTECIMIENTO MÚLTIPLE EN UNA EMPRESA METALMECÁNICA
}

\author{
Verónica Aída MORTARA* \\ https://orcid.org/0000-0003-2387-2963 \\ LUCIANA BELÉN TABONE* \\ https://orcid.org/0000-0003-3755-5336
}

Facultad de Ingeniería, Universidad Nacional de Mar del Plata, Argentina

Recibido: 16 de octubre del 2020 / Aprobado: 23 de noviembre del 2020

doi: https://doi.org/10.26439/ing.ind2021.n40.4809

RESUMEN: Para mantenerse competitivas, las organizaciones necesitan forjar relaciones estratégicas y colaborativas en su cadena de suministro. El objetivo del trabajo es diseñar un método de selección de proveedores bajo un entorno de abastecimiento múltiple. La metodología propuesta combina el proceso analítico de jerarquías y la programación por metas. En su aplicación, resulta indispensable la colaboración de los actores involucrados. El modelo obtiene la mejor combinación de proveedores, las cantidades a pedir a cada uno y permite sistematizar el proceso de selección en consistencia con la estrategia empresarial y las prioridades competitivas.

PALABRAS CLAVE: selección de proveedores / abastecimiento / proceso analítico de jerarquías / programación por metas

SUPPLIER SELECTION UNDER A MULTI-SOURCING STRATEGY

IN A METALWORKING COMPANY

ABSTRACT: To stay competitive, organizations need strategic and collaborative relationships with their supply chain. This research aims to design a method for supplier selection under a multi-sourcing environment. The proposed methodology combines the analytic hierarchy process and goal programming. The collaboration of the actors involved is essential for its application. The model selects the best combination of suppliers and the quantities to order from each one. It also allows systematizing the supplier selection process in accordance with the business strategy and competitive priorities.

KEYWORDS: supplier selection / sourcing / analytic hierarchy process / goal programming

*Correos electrónicos: vmortara@fi.mdp.edu.ar, ltabone@fi.mdp.edu.ar 


\section{INTRODUCCIÓN}

La creciente competitividad y la globalización exigen respuestas eficientes por parte de las empresas, como la definición de estrategias que les permitan sobrevivir en un mundo en continuo cambio, por lo que el desarrollo de estrategias competitivas basadas en la agregación de valor y la reducción simultánea de costos son objetivos ineludibles. En la gestión de la cadena de suministro, se considera al aprovisionamiento como una función estratégica de alto impacto en la productividad y el alcance de ventajas competitivas sostenibles para las empresas. Es importante la generación de integraciones verticales mediante modelos de colaboración con los involucrados en los procesos de la organización (Valencia, Cardona y Álvarez, 2018). Un aspecto para considerar es que los principales factores relacionados con el éxito en la integración de las cadenas de suministro son el recurso humano, las estrategias de la organización, la información y las tecnologías de la información implementadas (Bustillos Arizmendi y Carballo Mendívil, 2018).

Un adecuado diseño de la cadena de suministro es el punto de partida para definir la base de proveedores que responda a las prioridades competitivas de la función de producción u operaciones, la estrategia de la cadena de suministro, su nivel de integración y el rendimiento de la empresa (Qi, Huo, Wang y Yeung, 2017). De esta manera, la selección de los proveedores se ha convertido en una actividad crítica para las organizaciones que afecta su eficiencia y rentabilidad (Mendoza, Santiago y Ravi Ravindran, 2008).

El presente trabajo aborda un estudio de caso en el área de compras de una empresa metalmecánica que se dedica a la fabricación y comercialización de equipos de uso industrial y comercial. Sus prioridades competitivas son la personalización, la entrega a tiempo y la calidad superior. La empresa certificó desde el año 2006 su sistema de gestión de calidad bajo la norma ISO 9001. Del análisis de sus productos se pudo comprobar que un $20 \%$ de sus componentes comprados fueron considerados críticos para el normal funcionamiento de los equipos. En particular, se analizó la compra de uno de los componentes críticos, ya que todos los equipos requieren una unidad del mismo La empresa se abastece de dicho componente mediante cinco proveedores que se encuentran aprobados por su sistema de gestión de calidad. Su disponibilidad y correcto funcionamiento fueron determinantes al momento de evaluar la calidad del equipo por parte del cliente. Este componente puede ser adquirido a través de múltiples proveedores, por ello la selección de los mismos y las cantidades a comprar a cada uno se ha convertido en un proceso clave para el área de compras de la organización porque afecta directamente su productividad y sostenibilidad futura.

El objetivo fue diseñar una metodología dinámica e imparcial que permitiera seleccionar, en forma sistemática, los mejores proveedores de componentes en un entorno de abastecimiento múltiple. El término "mejor" significa que las características de los 
proveedores deberán ajustarse a las prioridades competitivas de la organización, que se traducen en los criterios de selección establecidos. Asimismo resultó fundamental que la organización estableciera relaciones colaborativas con sus proveedores donde ambos pudieran beneficiarse del intercambio mutuo, no solo en términos de rentabilidad, sino también a favor de la mejora del desempeño y la sostenibilidad de la cadena global y satisfacción del cliente final.

Este trabajo se organizó en las siguientes secciones: una introducción que detalla el caso de estudio y problemática abordada, luego se presenta una revisión de la literatura con los principales antecedentes teóricos y prácticos del área de estudio, se continúa con la especificación de la metodología propuesta y, finalmente, se exponen los resultados obtenidos, discusión y conclusiones más relevantes.

\section{REVISIÓN DE LA LITERATURA}

Las exigencias del entorno competitivo actual han generado que la función de aprovisionamientos deje de ser considerada, únicamente, como una operación de compra y administración del inventario, para pasar a reconocer su importancia en la estrategia empresarial (Burt, Dobler y Starling, 2003). Por ello diversos autores consideran que la fiabilidad, la calidad y el nivel de desempeño de una empresa dependen, en gran parte, del desempeño de su base de proveedores (Ghodsypour y O'Brien, 2001; Sarache Castro, Castrillón y Ortiz Franco, 2009; Kamman y Bakker, 2004; Nuñez-Perez et al., 2017).

La selección de proveedores, como parte del proceso de abastecimiento, se ha convertido en un proceso de decisión clave para toda organización. La definición de la metodología y criterios a evaluar para su correcta selección se han vuelto una tarea compleja y dificultosa (Herrera Umaña y Osorio Gómez, 2006; Ortiz Torres, Márquez Sánchez, Oramas Santos y Marrero Ancízar, 2018; Escandón López, Parra Calderón y Osorio Gómez, 2019). Esto radica en el número de alternativas y criterios asociados y es por ello la vital importancia que reviste la selección de las técnicas adecuadas que permitan responder a los múltiples criterios de decisión y a la selección de las mejores alternativas de forma global.

Para la adecuada selección de proveedores es necesario evaluar un conjunto de factores muy amplios y se deben tener en cuenta criterios cualitativos y cuantitativos (Ghodsypour y O'Brien, 1998; de Boer, Labro y Morlacchi, 2001; Liu y Hai 2005). Schroeder, Anderson y Cleveland (1986) establecen que el costo, la flexibilidad, la calidad y la entrega son factores básicos a evaluar; por otro lado, Weber, Current y Benton (1991) plantean que los más importantes son la calidad y la entrega; Miltenburg (1995) detecta la aparición de uno nuevo que es el servicio; Modi y Mabert (2006) resaltan la importancia de la calidad y el costo. 
Respecto al tamaño de la base de proveedores, Ghodsypour y O'Brien (1998) plantean que el abastecimiento puede ser con fuente única o múltiple. En el primer caso, un único proveedor está capacitado para satisfacer el conjunto de criterios de selección de forma satisfactoria, mientras que, en el segundo, se tienen varias opciones a elegir. Dowlatshahi (2000), Ghodsypour y O'Brien (2001) y Choy, Lee y Lo (2003) plantean que cuando se trata del surtido de pedidos de materiales o insumos considerados críticos para la organización resulta beneficioso el uso de fuentes de abastecimiento múltiples. Esto se debe a que el desabastecimiento de un material crítico puede ocasionar inconvenientes en el proceso productivo y contar con al menos dos proveedores capaces de suministrarlo logra reducir este riesgo (Ortiz Torres, 2018).

Analizando las técnicas a utilizar, estas pueden ser individuales o integradas, las últimas son el resultado de la combinación de dos o más técnicas. Las técnicas multicriterio son ampliamente utilizadas para la evaluación de proveedores ya que permiten disminuir la subjetividad asociada al proceso de selección. Esto garantiza que el mejor proveedor sea el que más se ajuste a los criterios requeridos y que se disminuya la incertidumbre en la elección final (Ortiz Torres et al., 2018; Escandón López, 2019). Sin embargo, resulta necesario su combinación con técnicas más exactas para definir las cantidades de compra a cada proveedor en escenarios de múltiple abastecimiento (Narasimhan, Talluri y Méndez, 2001). De Boer et al. (2001), Ghodsypour y O'Brien (2001), Ganeshan, Tyworth y Guo (1999) consideran la programación matemática como una herramienta beneficiosa para la toma de esta decisión.

Las técnicas individuales más aplicadas son el proceso analítico de jerarquías, los procesos de red analítica, el análisis de envolvente de datos, la lógica difusa, los algoritmos genéticos, la programación lineal, la programación por metas, la técnica de calificación de atributos múltiples sencillos, el razonamiento basado en casos, entre otros métodos (F. T. Chan y H. K. Chan, 2004; Ding, Benyoucef y Xie, 2005; Talluri y Narasimhan, 2005; Liu y Hai, 2005; K. L. Choy, Lee, Lau y L. C. Choy, 2005; Talluri, Narasimhan y Nair, 2006; Garfamy, 2006; Chen, Lin y Huang, 2006; Huang y Keskar, 2007; Ho, Xu y Dey, 2010; Dalalah, Hayajneh y Batieha, 2011; Ortiz Torres, Oramas Santos, Sanz Pérez, 2015).

En el uso de técnicas integradas al proceso analítico de jerarquías es el más combinado. Ha sido aplicado con lógica difusa, programación por metas, análisis de envolvente de datos y razonamiento basado en casos (Kahraman, Cebeci y Ulukan, 2003; Perçin, 2006; Curkovic y Handfield, 2006; Șevkli, Lenny Koh, Zaim, Demirbag y Tatoglu, 2007; Ortiz Torres et al., 2018).

Según Sarache Castro et al. (2009), no existe un único método a aplicarse en el proceso de selección de proveedores, pero existe una tendencia en emplear, primero, un método de aproximación y, luego, refinar la decisión final con un método más exacto. 


\section{METODOLOGÍA}

La metodología adoptada consistió en un estudio de caso, desarrollado en una empresa metalmecánica de la ciudad de Mar del Plata, de carácter empírico, puesto que se estudió un fenómeno contemporáneo dentro de su entorno. La investigación fue de tipo mixta, cualitativa y cuantitativa. Inicialmente, se analizaron datos en forma descriptiva y luego, mediante la aplicación de las herramientas propuestas, se realizaron cálculos matemáticos para obtener las priorizaciones y selección final de proveedores.

Los pasos que se aplicaron en este trabajo fueron los siguientes:

a) Realización de entrevistas al gerente y responsable de compras de la organización para conocer las características y necesidades de la misma, visitas in situ para comprender el funcionamiento de la empresa y las particularidades del sistema de compras.

b) Recolección de información relevante sobre las necesidades del sistema de compra para la definición de criterios y subcriterios de selección de proveedores, en función de las prioridades competitivas del área productiva y desempeño de los proveedores para su posterior análisis.

c) Implementación del proceso analítico de jerarquías (PAJ) para la priorización de los mismos mediante entrevistas al gerente y responsable de compras. Toda la información recopilada se procesó en el software Expert Choice ${ }^{\circledR}$.

d) Selección final de proveedores y determinación de las cantidades a pedir a cada uno mediante el diseño de un modelo de programación por metas (PM). El procesamiento se efectuó con el software $\mathrm{QM}^{\circledR}$.

Análisis de resultados a partir de la aplicación de las herramientas propuestas.

Los criterios y subcriterios de selección de proveedores se definieron en forma precisa, medible y comparable. En la tabla 1 se especifican los indicadores utilizados para su medición. 
Tabla 1

Criterios de selección de proveedores

\begin{tabular}{lcc}
\hline Criterios & Subcriterios & Indicadores \\
\hline Servicio & Garantía & Años \\
& Capacidad del proceso & Índice $C_{p k}$ \\
Nivel de servicio & Porcentaje de entregas a tiempo \\
Calidad & Defectos & Porcentaje de partes defectuosas \\
Flexibilidad & & Porcentaje \\
Precio & & Dólares \\
Entrega & Distancia & Kilómetros \\
& Tiempo de entrega & Días/parte \\
\hline
\end{tabular}

Elaboración propia

\subsection{Proceso analítico de jerarquías (PAJ)}

La jerarquización de proveedores mediante el PAJ permite tomar decisiones en base a criterios múltiples y trabaja en forma sencilla, lógica y estructurada (Martínez Rodríguez, 2007). Se basa en la disgregación del problema complejo en una estructura jerárquica multinivel de objetivos o metas, criterios, subcriterios y alternativas (Xu y Liao, 2014). El proceso requiere que quien toma las decisiones proporcione evaluaciones objetivas y/o subjetivas, respecto a la importancia relativa de cada uno de los criterios que se seguirán para tomar la decisión, especificando su preferencia con respecto a cada una de las alternativas de decisión referida a cada criterio (Toskano Hurtado, 2005). El resultado del PAJ muestra la prioridad total de las alternativas de decisión respecto a la meta (Saaty, 2008). En esta etapa, se obtuvo un ordenamiento de los proveedores a partir de la ponderación global de los mismos y para su desarrollo se utilizó el software Expert Choice $^{\circledR}$.

Para el desarrollo del PAJ se efectuaron los siguientes pasos (Saaty, 2008):

a) Definición del problema, estableciendo sus componentes relevantes.

b) Construcción de la estructura jerárquica del problema mediante su representación gráfica en función de la meta global, los criterios y subcriterios y las alternativas de decisión (figura 1). 


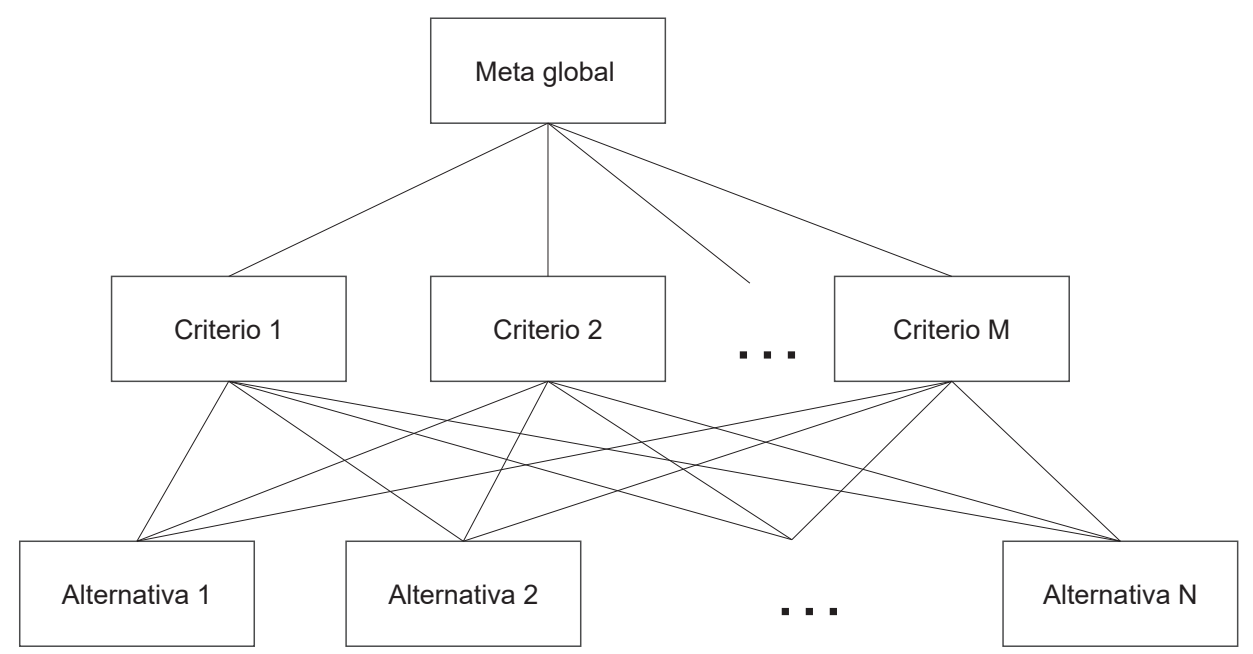

Fuente: Toskano Hurtado (2005)

Elaboración propia

c) Establecimiento de las preferencias: los decisores expresaron su juicio de valor con respecto a todos los elementos de un nivel, referido a cada uno de los elementos del nivel inmediato superior. Para establecer dichas preferencias, se utilizaron matrices de comparaciones pareadas y para su construcción se empleó la escala de Saaty con valores de 1 a 9, como se muestra en tabla 2, para calificar la importancia relativa de los elementos. Esta escala de calificación permitió la disminución del grado de subjetividad, en la evaluación de los factores cualitativos (Salas Bacalla, Leyva Caballero y Calenzani Fiestas, 2014).

d) Priorización y sintetización: mediante un proceso matemático se calcularon las prioridades relativas locales de cada elemento para cada nodo de la estructura jerárquica. Se realizó un análisis de la calidad de decisión final, examinando la consistencia de juicios de los decisores. Se calculó la relación o razón de consistencia de Saaty, considerando aceptable los valores menores a 0,1. Utilizando el principio de composición jerárquica, se obtuvieron las prioridades globales de los criterios para cada nodo respecto de la meta. Por último, se realizó la síntesis del problema para obtener la prioridad total de las alternativas mediante la agregación multiaditiva de las prioridades globales. Esto permitió ordenar el conjunto de las alternativas consideradas y seleccionar la mejor para obtener la meta buscada. 
Tabla 2

Escala de Saaty

\begin{tabular}{cll}
\hline Puntaje & \multicolumn{1}{c}{ Definición } & \multicolumn{1}{c}{ Explicación } \\
\hline 1 & Igual importancia & Los dos elementos contribuyen igualmente al objetivo. \\
3 & Importancia moderada & $\begin{array}{l}\text { La experiencia y el juicio ligeramente a favor de uno de los } \\
\text { elementos. }\end{array}$ \\
5 & Importancia fuerte & $\begin{array}{l}\text { La experiencia y el juicio fuertemente a favor de uno de los } \\
\text { elementos }\end{array}$ \\
7 & $\begin{array}{l}\text { Importancia muy fuerte } \\
\text { o demostrable }\end{array}$ & $\begin{array}{l}\text { Un elemento es preferido sobre el otro en un grado muy fuerte } \\
\text { y esta preferencia puede demostrarse en la práctica. }\end{array}$ \\
& Importancia extrema & $\begin{array}{l}\text { La evidencia favorece a una alternativa sobre la otra } \\
\text { extremadamente. }\end{array}$ \\
& Para valores inter- & Algunas veces se necesita interpolar un juicio, porque no hay \\
medios entre las & una palabra que describa la relación entre los elementos. \\
\hline
\end{tabular}

Fuente: Saaty (2008)

Elaboración propia

\subsection{Programación por metas (PM)}

La selección final de proveedores a través de PM es de utilidad para los tomadores de decisiones porque permite discutir y encontrar un conjunto de soluciones apropiadas y aceptables en problemas de decisión con objetivos o criterios múltiples (H. A. López Ospina y R. D. López Ospina, 2010).

La PM posee gran potencialidad cuando el contexto decisional está definido por una serie de objetivos a optimizar que deben de satisfacer un conjunto de restricciones. Al considerarse prácticamente imposible alcanzar simultáneamente varios objetivos, porque entran en conflicto, el decisor se conforma con fijar una meta para cada uno, intentando minimizar la falta de logro de las mismas (Jiménez López, 2012). De esta manera, la PM en vez de buscar un punto óptimo pretende determinar un conjunto de soluciones eficientes u óptimas (López, Fernández Henao y Morales, 2007).

El modelo de PM propuesto fue una adaptación del presentado por Mendoza et al. (2008) y Mohile (2008). Las modificaciones se realizaron para atender las características particulares de la organización en estudio y en función de sus prioridades competitivas. En la tabla 3 se presentan las constantes y variables que se utilizaron. 
Tabla 3

Nomenclatura de los conceptos

\begin{tabular}{|c|c|}
\hline Nomenclatura & Concepto \\
\hline N & Cantidad de proveedores \\
\hline$x_{i}$ & Cantidad pedida al proveedor $i$ \\
\hline$D$ & Demanda anual \\
\hline$g_{i}$ & Garantía del proveedor $i$ \\
\hline$C_{i}$ & Capacidad del proveedor $i$ \\
\hline$P G_{i}$ & Ponderación global del proveedor $i$ \\
\hline$T E_{i}$ & Tiempo de entrega requerido para el proveedor $i$ \\
\hline te & Tiempo de entrega del proveedor $i$ para suministrar una unidad de producto \\
\hline pk & Nivel de $C_{p k}$ requerido \\
\hline$C_{\rho i}$ & $\mathrm{C}_{\mathrm{pk}}$ del proveedor $i$ \\
\hline$q_{i}$ & Defectos del proveedor $i$ (porcentaje) \\
\hline G & Garantía requerida \\
\hline NS & Nivel de servicio requerido \\
\hline$n s_{i}$ & Nivel de servicio del proveedor $i$ \\
\hline F & Nivel de flexibilidad requerida \\
\hline$f_{i}$ & Nivel de flexibilidad del proveedor $i$ \\
\hline$P_{i}$ & Precio del proveedor $i$ \\
\hline$K_{i}$ & Distancia del proveedor $i$ al comprador \\
\hline$Y_{i}$ & 1, si una orden es asignada al proveedor $i$; 0 , si no es asignada \\
\hline$d^{+}$ & Sobrelogro de la meta \\
\hline$d^{-}$ & Sublogro de la meta \\
\hline
\end{tabular}

Elaboración propia

Las restricciones de la meta se definieron en función de los criterios de selección especificados previamente y se presentan en las ecuaciones (1) a (9). Las restricciones estructurales se muestran en las ecuaciones (10) a (13).

- Restricciones de la meta

Valor de compra ponderado: surge de los resultados del PAJ para ponderar las cantidades a pedir de acuerdo con la $P G$ obtenida. Se maximizó este valor, considerando un valor relativamente grande, $M$.

$$
\sum_{i=1}^{n} P G_{i} X_{i}+d_{1}^{-}-d_{1}^{+}=M
$$


Garantía: es la cantidad de años de garantía otorgada por el proveedor.

$$
\sum_{i=1}^{n} g_{i} Y_{i}+d_{2}^{-}-d_{2}^{+}=G \sum_{i=1}^{n} Y_{i}
$$

Capacidad de proceso: representa el nivel de capacidad de abastecimiento del proveedor con respecto a una capacidad crítica.

$$
\sum_{i=1}^{n} C_{p i} Y_{i}+d_{3}^{-}-d_{3}^{+}=\overline{C_{p k}} \sum_{i=1}^{n} Y_{i}
$$

Nivel de servicio global: se refiere al porcentaje de entregas realizadas a tiempo.

$$
\sum_{i=1}^{n} n s_{i} Y_{i}+d_{4}^{-}-d_{4}^{+}=N S \sum_{i=1}^{n} Y_{i}
$$

Calidad: considera el porcentaje de defectos aceptable.

$$
\sum_{i=1}^{n} q_{i} Y_{i}+d_{5}^{-}-d_{5}^{+}=0
$$

Flexibilidad global: permite que la compañía responda fácilmente a los cambios de la demanda.

$$
\sum_{i=1}^{n} f_{i} Y_{i}+d_{6}^{-}-d_{6}^{+}=F \sum_{i=1}^{n} Y_{i}
$$

Gastos de compra: es el costo de aprovisionamiento del pedido.

$$
\sum_{i=1}^{n} P_{i} X_{i}+d_{7}^{-}-d_{7}^{+}=0
$$

Distancia total: considera la distancia total de los proveedores seleccionados a la empresa.

$$
\sum_{i=1}^{n} K_{i} Y_{i}+d_{8}^{-}-d_{8}^{+}=0
$$


Tiempo de entrega: es el tiempo que el proveedor requiere para realizar la entrega.

$t e_{i} X_{i}+d_{j}^{-}-d_{j}^{+}=T E_{i} \quad i=1,2, \ldots, n ; j=9,9+1, \ldots, 9+n$

- Restricciones estructurales

$$
\begin{gathered}
\sum_{i=1}^{n} X_{i}=D \\
X_{i} \leq C_{i} Y_{i} \quad i=1,2, \ldots, n \\
\sum_{i=1}^{n} Y_{i} \leq n
\end{gathered}
$$

Luego se establecieron las prioridades de cada meta y la meta global según las características de la empresa estudiada. Finalmente, se realizó un análisis de sensibilidad variando el orden de las prioridades.

\section{RESULTADOS}

\subsection{Jerarquización de proveedores}

La estructura jerárquica para el desarrollo del PAJ se presenta en la figura 2 . Se puede observar que la meta consiste en la selección del mejor proveedor para el componente crítico. Las alternativas planteadas fueron los cinco proveedores aprobados por el sistema de gestión de calidad de la empresa y la selección se realizó en base a los criterios y subcriterios establecidos en la tabla 1. 


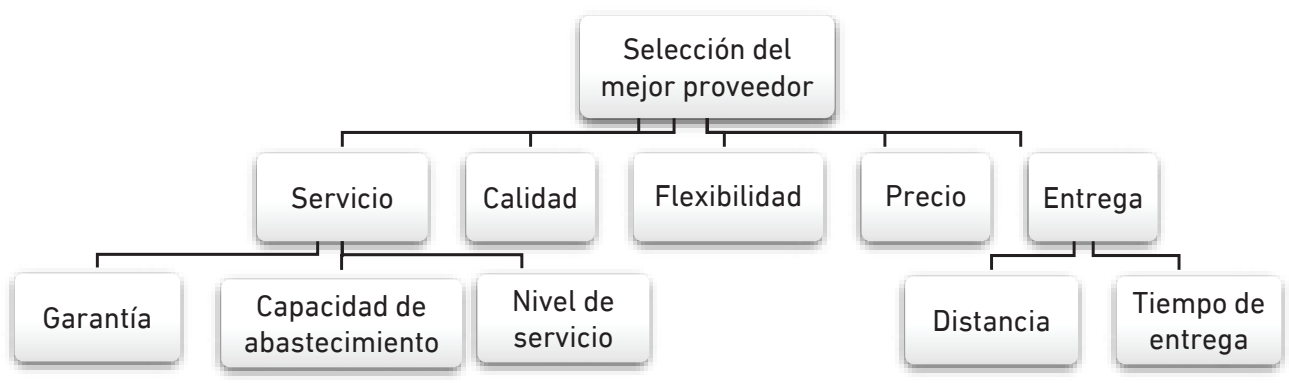

Figura 2. Árbol de jerarquía PAJ

Elaboración propia

Para la construcción de las matrices se utilizaron los valores de los indicadores que se presentan en la tabla 4. A modo de ejemplo, en la tabla 5 se muestran la matriz de comparación pareada y los pesos obtenidos para los criterios de selección. Se observa que la entrega, la flexibilidad y la calidad, en ese orden, son las características más importantes en el proceso de selección de proveedores de la empresa.

Tabla 4

Valor de indicadores por proveedor

\begin{tabular}{|c|c|c|c|c|c|c|c|c|c|}
\hline $\begin{array}{l}\grave{0} \\
0 \\
0 \\
0 \\
0 \\
0 \\
0\end{array}$ & 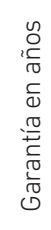 & $\begin{array}{l}\frac{0}{0} \\
\frac{\pi}{0} \\
\frac{0}{0} \\
\frac{0}{0} \\
0 \\
0\end{array}$ & 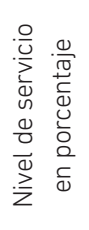 & 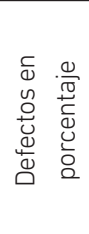 & 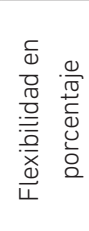 & 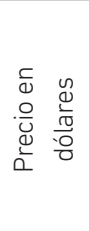 & 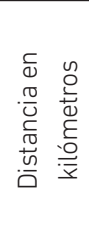 & 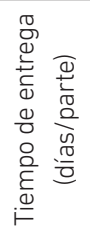 & 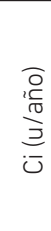 \\
\hline 1 & 1 & 0,8 & 75 & 5 & 10 & 306 & 475 & 30 & 200 \\
\hline 2 & 2 & 1 & 90 & 1 & 5 & 847 & 2770 & 45 & 200 \\
\hline 3 & 2 & 0,85 & 95 & 2 & 2 & 308 & 460 & 10 & 180 \\
\hline 4 & 1 & 0,9 & 98 & 1 & 5 & 647 & 575 & 15 & 150 \\
\hline 5 & 2 & 0,83 & 80 & 3 & 0 & 651 & 713 & 20 & 180 \\
\hline $\begin{array}{l}\text { Valores } \\
\text { ideales }\end{array}$ & 2 & 1 & 100 & 1 & 20 & 300 & 20 & 10 & 350 \\
\hline
\end{tabular}

Elaboración propia 
Tabla 5

Matriz de comparación pareada y pesos de criterios de selección

\begin{tabular}{lcccccc}
\hline & Precio & Calidad & Flexibilidad & Servicio & Entrega & Pesos \\
\hline Precio & 1 & $1 / 8$ & $1 / 9$ & 2 & $1 / 8$ & 0,045 \\
Calidad & 8 & 1 & $1 / 2$ & 4 & $1 / 2$ & 0,208 \\
Flexibilidad & 9 & 2 & 1 & 6 & $1 / 2$ & 0,301 \\
Servicio & $1 / 2$ & $1 / 4$ & $1 / 6$ & 1 & $1 / 8$ & 0,041 \\
Entrega & 8 & 2 & 2 & 8 & 1 & 0,404 \\
\hline
\end{tabular}

Elaboración propia

Luego, se procesó toda la información en el software Expert Choice ${ }^{\circledR}$ y se obtuvo la prioridad global de cada proveedor según se muestra en la figura 3. De esta manera, se generó la siguiente jerarquización: proveedor 4 , proveedor 3 , proveedor 1, proveedor 2 y proveedor 5. Los índices de consistencia obtenidos fueron aceptables para todos los juicios realizados.

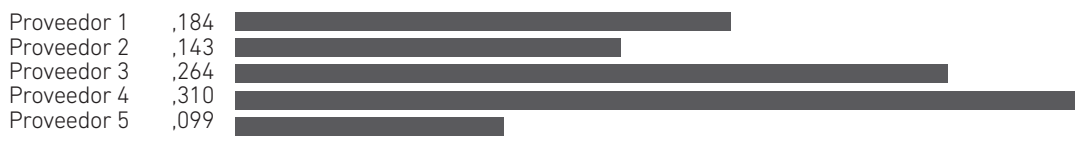

Figura 3. Puntaje global de cada proveedor

Elaboración propia en Expert Choice ${ }^{\circledast}$

\subsection{Selección de proveedores}

Para determinar los proveedores a seleccionar y las cantidades a comprar a cada uno, se aplicó el modelo de PM propuesto y presentado en las ecuaciones (1) a (12).

En la tabla 5 se establecen las prioridades de las metas del modelo en función de la importancia de los criterios considerados en la etapa anterior y se define la siguiente función objetivo (ecuación 13):

$$
\begin{aligned}
\operatorname{Min} Z=P_{1}\left(d_{1}^{-}\right) & +P_{2}\left(d_{6}^{-}+d_{9}^{+}+d_{10}^{+}+d_{11}^{+}+d_{12}^{+}+d_{13}^{+}\right) \\
& +P_{3}\left(d_{5}^{+}+d_{7}^{+}\right)+P_{4}\left(d_{2}^{-}+d_{4}^{-}\right)+P_{5}\left(d_{8}^{+}\right) \\
& +P_{6}\left(d_{3}^{-}\right)
\end{aligned}
$$

Para la ecuación (1) se utilizaron los valores de prioridad global obtenidos en el PAJ. Los datos que se ingresaron a las ecuaciones (2) a (8) del modelo son los presentados en 
la tabla 6. El valor de la demanda anual (D) en la ecuación (9) es 750 partes, que debe ser satisfecha, en su totalidad, por un único proveedor o una combinación de ellos.

Tabla 6

Prioridades del modelo de PM

\begin{tabular}{cll}
\hline Prioridad & \multicolumn{1}{c}{ Restricción de la meta } & Desviaciones que controlar \\
\hline P1 & Valor de compra ponderado & $\mathrm{d} 1-$ \\
P2 & Flexibilidad y tiempo de entrega & $\mathrm{d} 6-, \mathrm{d}$ 9+, d10+, d11+, d12+, d13+ \\
P3 & Calidad y gastos de compra & $\mathrm{d} 5+, \mathrm{d} 7+$ \\
P4 & Garantía y nivel de servicio & $\mathrm{d} 2-, \mathrm{d} 4-$ \\
P5 & Distancia & $\mathrm{d} 8+$ \\
P6 & Capacidad & $\mathrm{d} 3-$ \\
\hline
\end{tabular}

Elaboración propia

El resultado del modelo de $\mathrm{PM}$ obtenido mediante el software $\mathrm{QM}^{\circledR}$ permitió determinar que los proveedores seleccionados son el 1, 3 y 4 y se les debería comprar 255, 270 y 225 partes a cada uno, respectivamente. Los proveedores 2 y 5 quedaron fuera de la selección.

\subsection{Análisis de sensibilidad}

Para evaluar la robustez del modelo, se plantearon y analizaron cinco escenarios alternativos (E2 a E6) a la situación real (E1), cambiando el orden de las prioridades en la función objetivo. De los seis escenarios posibles, se seleccionaron aquellos que fueron más representativos de las necesidades del sector industrial. Los escenarios planteados y sus resultados se muestran en la tabla 7 y 8 . 
Tabla 7

Análisis de escenarios

\begin{tabular}{|c|c|c|c|c|c|c|}
\hline & E1 & E2 & E3 & E4 & E5 & E6 \\
\hline P1 & $\begin{array}{l}\text { Valor de } \\
\text { compra } \\
\text { ponderado }\end{array}$ & $\begin{array}{l}\text { Flexibilidad } \\
\text { y tiempo de } \\
\text { entrega }\end{array}$ & $\begin{array}{l}\text { Calidad y } \\
\text { gastos de } \\
\text { compra }\end{array}$ & $\begin{array}{c}\text { Valor de } \\
\text { compra } \\
\text { ponderado }\end{array}$ & Distancia & $\begin{array}{l}\text { Valor de com- } \\
\text { pra ponderado }\end{array}$ \\
\hline P2 & $\begin{array}{c}\text { Flexibilidad } \\
\text { y tiempo de } \\
\text { entrega }\end{array}$ & $\begin{array}{l}\text { Valor de } \\
\text { compra } \\
\text { ponderado }\end{array}$ & $\begin{array}{c}\text { Valor de } \\
\text { compra } \\
\text { ponderado }\end{array}$ & $\begin{array}{l}\text { Calidad y } \\
\text { gastos de } \\
\text { compra }\end{array}$ & $\begin{array}{c}\text { Valor de compra } \\
\text { ponderado }\end{array}$ & $\begin{array}{l}\text { Garantía y ni- } \\
\text { vel de servicio }\end{array}$ \\
\hline P3 & $\begin{array}{l}\text { Calidad y } \\
\text { gastos de } \\
\text { compra }\end{array}$ & $\begin{array}{l}\text { Calidad y gas- } \\
\text { tos de compra }\end{array}$ & $\begin{array}{c}\text { Flexibilidad } \\
\text { y tiempo de } \\
\text { entrega }\end{array}$ & $\begin{array}{c}\text { Flexibilidad } \\
\text { y tiempo } \\
\text { de entrega }\end{array}$ & $\begin{array}{c}\text { Flexibilidad y tiem- } \\
\text { po de entrega }\end{array}$ & $\begin{array}{c}\text { Flexibilidad } \\
\text { y tiempo de } \\
\text { entrega }\end{array}$ \\
\hline P4 & $\begin{array}{l}\text { Garantía } \\
\text { y nivel de } \\
\text { servicio }\end{array}$ & $\begin{array}{l}\text { Garantía y ni- } \\
\text { vel de servicio }\end{array}$ & Distancia & $\begin{array}{l}\text { Garantía } \\
\text { y nivel de } \\
\text { servicio }\end{array}$ & $\begin{array}{c}\text { Calidad y gastos de } \\
\text { compra }\end{array}$ & $\begin{array}{l}\text { Calidad y gas- } \\
\text { tos de compra }\end{array}$ \\
\hline P5 & Distancia & Capacidad & Capacidad & Distancia & Capacidad & Distancia \\
\hline P6 & Capacidad & Distancia & $\begin{array}{l}\text { Garantía } \\
\text { y nivel de } \\
\text { servicio }\end{array}$ & Capacidad & $\begin{array}{c}\text { Garantía y nivel de } \\
\text { servicio }\end{array}$ & Capacidad \\
\hline
\end{tabular}

Elaboración propia

Tabla 8

Resultados obtenidos

\begin{tabular}{ccccccc}
\hline & E1 & E2 & E3 & E4 & E5 & E6 \\
\hline X1 & 255 & 0 & 300 & 255 & 255 & 255 \\
X2 & 0 & 0 & 0 & 0 & 0 & 0 \\
X3 & 270 & 270 & 270 & 270 & 270 & 270 \\
X4 & 225 & 225 & 180 & 225 & 225 & 225 \\
X5 & 0 & 255 & 0 & 0 & 0 & 0 \\
\hline
\end{tabular}

Elaboración propia

El análisis de sensibilidad demostró que la mezcla de proveedores seleccionados y las cantidades a comprar a cada uno no presentaron gran variación respecto al escenario 1, que representó la situación real del sistema de compras. 


\section{DISCUSIÓN}

La metodología propuesta resultó adecuada para la selección de proveedores ya que, como indican Sarache Castro et al. (2009), toma en consideración el análisis del contexto y las particularidades de la cadena de abastecimiento, la estrategia para la función de aprovisionamiento, los criterios de decisión y los métodos de selección como apoyo a la decisión.

Respecto a los criterios y las metas definidas, se encontraron en concordancia con los planteamientos realizados por diversos autores a lo largo de las últimas décadas. Para el proceso de selección, no solo se consideró el costo, la flexibilidad y la entrega, sino que se tuvo en cuenta también la calidad y el servicio ofrecido.

En los trabajos de Masella y Rangone (2000), Liu y Hai (2005), Chiang y Bai (2006), Osorio Gómez et al. (2008), Medina Varela et al. (2012) y Cordero Torres (2019) se aplica el PAJ en la gestión de proveedores, demostrando su capacidad para integrar sistemáticamente los juicios de los expertos, obtener el peso de los criterios de evaluación y determinar los mejores proveedores que responden a las necesidades de la empresa. Diversos autores (Ghodsypour y O'Brien, 1998; de Boer et al., 2001; Narasimhan et al., 2001; Yang y Chen, 2006; Perçin, 2006; Kokangul y Susuz, 2009; Ortiz Torres et al., 2018) plantean que para obtener la decisión final de cuánto comprar a cada proveedor, se debe utilizar, además, alguna técnica más exacta, como son los modelos matemáticos. En línea con estos, el método propuesto utilizó la PM para obtener las cantidades finales a adquirir de cada proveedor, a partir de los resultados obtenidos de la aplicación del PAJ.

\section{CONCLUSIONES}

La primera etapa de la metodología propuesta, PAJ, permitió la estructuración del problema de selección de proveedores en forma sencilla, teniendo en cuenta criterios de selección acordes a los objetivos estratégicos y a las prioridades competitivas del área de producción de la empresa en estudio. Se obtuvo una jerarquización de los proveedores considerados y se utilizaron las prioridades globales de cada uno como dato de entrada de la segunda etapa de esta metodología.

El modelo de PM propuesto obtuvo la mejor combinación de proveedores y las cantidades a pedir a cada uno. Este permite priorizar las metas o los criterios según las necesidades del proceso de selección de cada empresa. Finalmente, para el caso en estudio se comprobó la robustez de la solución obtenida mediante un análisis de sensibilidad. Este análisis arrojó que, de los seis escenarios analizados, cinco (83,33\%) presentaron la misma combinación de proveedores seleccionados y cuatro $(66,67 \%)$ obtuvieron las mismas cantidades a comprar a cada uno. 
La metodología propuesta le permitió al responsable de compras realizar el proceso de selección de proveedores en forma sistemática y consistente con la estrategia de la empresa y las prioridades competitivas del área productiva. La utilización de los softwares Expert Choice ${ }^{\circledR}$ y $\mathrm{QM}^{\circledR}$ resultaron ser sumamente sencilla y de fácil comprensión. Se concluye que la metodología propuesta permitió sistematizar el proceso de selección de proveedores en forma consistente con la estrategia de la empresa y las prioridades del área productiva. Es de viable implementación para la empresa ya que los datos de entrada son derivados de la actividad habitual del proceso de gestión de proveedores. Se comprueba que es apropiada para aplicar en el proceso de selección de proveedores porque permite obtener la mejor combinación de cantidades a comprar a cada proveedor considerando criterios acordes las características y necesidades de cada empresa.

Es condición necesaria el trabajo colaborativo, la comunicación efectiva y la confianza recíproca de todos los actores de la cadena de suministro interna y externa a la empresa para alanzar un eficiente intercambio de información que les permita mejorar su desempeño. En consecuencia, se espera un incremento de los niveles de conformidad del cliente final al poder brindarle una respuesta superadora en términos de calidad y tiempos de entrega.

La metodología propuesta reviste una especial contribución al análisis de las operaciones de aprovisionamiento, dado su carácter estratégico y el impacto que genera en el alcance de ventajas competitivas sostenibles. Como su aplicación requiere de una relación estrecha entre la empresa y sus proveedores, se potencia la posibilidad de integración de sus sistemas de información para poder, a futuro, agilizar el intercambio de información y, en consecuencia, el aumento de la velocidad del proceso de toma de decisiones relativas al aprovisionamiento. De esta manera, se puede lograr la transformación digital requerida en la nueva era de la industria 4.0 en la empresa y su cadena de suministros.

Como trabajo futuro, se considera fundamental el análisis de las tecnologías de la era analítica que puedan ser incorporadas en el proceso de abastecimiento de una organización para lograr una integración vertical eficiente en su cadena de suministros. Se reflexiona que esta transformación digital contribuirá a la supresión de los límites existentes entre los actores de la cadena, cambiando las relaciones sociales y económicas, conectando usuarios, sistemas y equipos y mejorando la productividad y la calidad de los procesos industriales.

\section{REFERENCIAS}

Burt, D. N., Dobler, D. W., y Starling, S. L. (2003). World class supply management: The key to supply chain management. Nueva York: McGraw-Hill. 
Bustillos Arizmendi, L. A., y Carballo Mendívil, B. (2018). Integración de la cadena de suministro: una revisión de la literatura. Revista Ingeniería Industrial, 17(3), 247-268.

Chan, F. T., y Chan, H. K. (2004). Development of the supplier selection model. A case study in the advanced technology industry. Proceedings of the Institution of Mechanical Engineers Part B: Journal of Engineering, 218(12), 1807-1824.

Chen, C. T., Lin, C. T., y Huang, S. F. (2006). A fuzzy approach for supplier evaluation and selection in supply chain management. International Journal of Production Economics, 102(2), 289-301.

Chiang, C., y Bai, S. (2006). Supplier selection using combined analytical hierarchy process and grey relational analysis. Journal of Manufacturing Technology Management, 17(7), 301-315.

Choy, K. L., Lee, W. B., Lau, H. C. W., y Choy, L. C. (2005). A knowledge-based supplier intelligence retrieval system for outsource manufacturing. Knowledge-Based Systems, 18(1), 1-17.

Choy, K., Lee, W., y Lo, V. (2003). Design of an intelligent supplier relationship management system: a hybrid case based neural network approach. Expert Systems with Applications, 24(2), 225-237.

Cordero Torres, B. (2019). Selección de proveedores priorizando criterios sostenibles para productos: un enfoque de AHP en compras públicas peruanas. Industrial Data, 22(1), 153-172. https://doi.org/10.15381/idata.v22i1.16532

Curkovic, S., y Handfield, R. B. (2006). Use of ISO 9000 and Baldrige Award Criteria in supplier quality evaluation. Journal of Supply Chain Management, 32(2), 2-11. doi:10.1111/j.1745-493X.1996.tb00220.x

Dalalah, D., Hayajneh, M., y Batieha, F. (2011). A fuzzy multi-criteria decision making model for supplier selection. Expert Systems with Applications, 38(7), 8384-8391. doi:10.1016/j.eswa.2011.01.031

De Boer, L., Labro, E., y Morlacchi, P. (2001). A review of methods supporting supplier selection. European Journal of Purchasing and Supply Management, 7(2), 75-89. doi:10.1016/S0969-7012(00)00028-9

Ding, H., Benyoucef, L., y Xie, X. (2005). A simulation optimization methodology for supplier selection problem. International Journal of Computer Integrated Manufacturing, 18(2-3), 210-224.

Dowlatshahi, S. (2000). Design-buyer-supplier interface: Theory versus practice. International Journal of Production Economics, 63(2), 111-130. https://doi. org/10.1016/S0925-5273(99)00009-2 
Escandón López, J. C., Parra Calderón, C. A., y Osorio Gómez, J. C. (2019). Metodología multicriterio para la selección de proveedores bajo consideraciones de riesgo. Scientia et Technica, 24(2), 232-239. https://doi.org/10.22517/23447214.19681

Ganeshan, R., Tyworth, J. E., y Guo, Y. (1999). Dual sourced supply chains: The discount supplier option. Transportation Research Part E: Logistics and Transportation Review, 35(1), 11-23. doi:10.1016/s1366-5545(98)00020-9.

Garfamy, R. M. (2006). A data envelopment analysis approach based on total cost of ownership for supplier selection. Journal of Enterprise Information Management 19(6), 662-678. https://doi.org/10.1108/17410390610708526.

Ghodsypour, S. H., y O'Brien, C. (1998). A decision support system for supplier selection using an integrated analytic hierarchy process and linear programming. International Journal of Production Economics, 56-57, 199-212. https://doi. org/10.1016/S0925-5273(97)00009-1

Ghodsypour, S. H., y O'Brien, C. (2001). The total cost of logistics in supplier selection, under conditions of multiple sourcing, multiple criteria and capacity constraint. International Journal of Production Economics, 73(1), 15-27. https://doi.org/10.1016/ S0925-5273(01)00093-7

Herrera Umaña, M. F., y Osorio Gómez, J. C. (2006). Modelo para la gestión de proveedores utilizando AHP difuso. Estudios Gerenciales, (99), 69-88.

Ho, W., Xu, X., y Dey, P. K. (2010). Multi-criteria decision making approaches for supplier evaluation and selection: A literature review. European Journal of Operational Research, 202(1), 16-24.

Huang, S. H., y Keskar, H. (2007). Comprehensive and configurable metrics for supplier selection. International Journal of Production Economics, 105(2), 510-523.

Jiménez López, M. (2012). Programación por metas con niveles de aspiración imprecisos. Revista de Dirección y Administración de Empresas, 19, 117-128.

Kahraman, C., Cebeci, U., y Ulukan, Z. (2003). Multi-criteria supplier selection using fuzzy AHP. Logistics Information Management, 16(6), 382-394. doi:10.1108/09576050310503367

Kamman, D. J. F., y Bakker, E. (2004). Changing supplier selection and relationship practices: A contagion process. Journal of Purchasing and Supply Management, 10(2), 55-64. doi:10.1016/j.pursup.2003.12.002

Kokangul, A., y Susuz, Z. (2009). Integrated analytical hierarch process and mathematical programming to supplier selection problem with quantity discount. Applied Mathematical Modelling, 33(3), 1417-1429. https://doi.org/10.1016/j. apm.2008.01.021 
Liu, F., y Hai, H. L. (2005). The voting analytic hierarchy process method for selecting supplier. International Journal of Production Economics, 97(3), 308-317. doi:10.1016/j.ijpe.2004.09.005

López Ospina, H. A., y López Ospina, R. D. (2010). Modelos de optimización por metas para el cálculo de estimadores en regresión múltiple. Ciencia e Ingeniería Neogranadina, 20(1), 133-157. https://doi.org/10.18359/rcin.285

López, J. F., Fernández Henao, S., y Morales, M. M. (2007). Aplicación de la programación por metas en la distribución de servicios entre empresas operadoras del sistema de transporte masivo. Scientia et Technica, 13(37), 339-343. https:// www.redalyc.org/pdf/849/84903758.pdf

Martínez Rodríguez, E. (2007). Aplicación del proceso jerárquico de análisis en la selección de la localización de una pyme. Anuario Jurídico y Económico Escurialense, (40), 523-542.

Masella, C. y Rangone, A. (2000). A contingent approach to the design of vendor selection systems for different types of cooperative customer/supplier relationships. International Journal of Operations \& Production Management, 20(1), 70-84. doi:10.1108/01443570010287044.

Medina Varela, P., Cruz Trejos, E., y Gómez Montoya, R. (2012). Selección de proveedor de WMS utilizando método AHP. Scientia et Technica, 17(52), 65-72.

Mendoza, A., Santiago, E., y Ravi Ravindran, A. (2008). A three-phase multicriteria method to the supplier selection problem. The International Journal of Industrial Engineering: Theory, Applications and Practice, 15(2), 195-210.

Miltenburg, J. (1995). Manufacturing strategy: How to formulate and implement a winning plan. Portland, Oregon: Productivity Press.

Modi, S. B., y Mabert, V. A. (2006). Supplier development: Improving supplier performance through knowledge transfer. Journal of Operations Management, 25(1), 42-64. doi:10.1016/j.jom.2006.02.001

Mohile, P. K. (2008). Multi-objetive decision on making for supplier selection in outsourcing (tesis de grado). Kate Gleason College of Engineering of the Rochester Institute of Technology, Nueva York, Estados Unidos.

Narasimhan, R., Talluri, S., y Méndez, D. (2001). Supplier evaluation and rationalization via data envelopment analysis. Journal of Supply Chain Management, 37(3), 28-37. doi:10.1111/j.1745-493X.2001.tb00103.x

Nuñez-Perez, N., Ortíz-Barrios, M., McClean, S., Salas-Navarro, K., Jimenez-Delgado, G., y Castillo-Zea, A. (2017). Discrete-event simulation to reduce waiting time in 
accident and emergency departments: A case study in a district general clinic. Lecture Notes in Computer Science, 10586, 352-363.

Ortiz Torres, M., Márquez Sánchez, F., Oramas Santos, O., y Marrero Ancízar, Y. (2018). Metodología para la evaluación de proveedores. Caso de estudio: empresa especializada importadora, exportadora y distribuidora para la ciencia y la técnica (EMIDICT). Revista Espacios, 39(27), 24-36.

Ortiz Torres, M., Oramas Santos, O., y Sanz Pérez, M. (2015). Procedimiento de evaluación de proveedores con herramientas de la teoría de los subconjuntos borrosos. Aplicación a proveedores seleccionados de una empresa comercial. Journal of Business, 7(1), 2-19.

Osorio Gómez, J. C., Herrera Umaña, M. F., y Vinasco, M. A. (2008). Modelo para la evaluación del desempeño de los proveedores utilizando AHP. Ingeniería y Desarrollo, (23), 43-58.

Perçin, S. (2006). An application of the integrated AHP-PGP model in supplier selection. Measuring Business Excellence, 10(4), 34-49. doi:10.1108/13683040610719263

Qi, Y., Huo, B., Wang, Z., y Yeung, H. Y. J. (2017). The impact of operations and supply chain strategies on integration and performance. International Journal of Production Economics, 185, 162-174.

Saaty, T. L. (2008). Decision making with the analytic hierarchy process. International Journal of Services Sciences, 1(1), 83-98. doi:10.1504/IJSSCI.2008.017590

Salas Bacalla, J., Leyva Caballero, M., y Calenzani Fiestas, A. (2014). Modelo del proceso jerárquico analítico para optimizar la localización de una planta industrial. Industrial Data, 17(2), 112-119. https://www.redalyc.org/pdf/816/81640856014.pdf

Sarache Castro, W. A., Castrillón, O. D., y Ortiz Franco, L. F. (2009). Selección de proveedores: una aproximación al estado del arte. Cuadernos de Administración, 22(38), 145-167.

Schroeder, R. G., Anderson, J. C., y Cleveland, G. (1986). The content of manufacturing strategy: An empirical study. Journal of Operations Management, 6(3-4), 405-415.

Şevkli, M., Lenny Koh, S. C., Zaim, S., Demirbag, M., y Tatoglu, E. (2007). An application of data envelopment analytic hierarchy process for supplier selection: a case study of BEKO in Turkey. International Journal of Production Research, 45(9), 1973-2003. doi:10.1080/00207540600957399

Talluri, S., y Narasimhan, R. (2005). A note on "A methodology for supply base optimization". IEEE Transactions on Engineering Management, 52(1), 130-139. doi:10.1109/ TEM.2004.839960 
Talluri, S. Narasimhan, R., y Nair, A. (2006). Vendor performance with supply risk: A chance-constrained DEA approach. International Journal of Production Economics, 100(2), 212-222. doi:10.1016/j.ijpe.2004.11.012

Toskano Hurtado, G. B. (2005). El proceso de análisis jerárquico (AHP) como herramienta para la toma de decisiones en la selección de proveedores (tesis de grado). Universidad Nacional Mayor San Marcos. Recuperada de http://sisbib.unmsm. edu.pe/bibvirtualdata/Tesis/Basic/toskano_hg/toskano_hg.pdf

Valencia, D. Cardona, C., y Álvarez, K. C. (2018). Mejoramiento del proceso de abastecimiento mediante Kraljic y Analytic Hierarchy Process - AHP en una empresa del sector textil. En E. Serna M. (Ed.), Investigación formativa en ingeniería (2. ${ }^{a}$ ed.) (pp. 236-251). Medellín: Editorial Instituto Antioqueño de Investigación.

Weber, C. A., Current, J. R., y Benton, W. C. (1991). Vendor selection criteria and methods. European Journal of Operation Research, 50(1), 2-18. https://doi.org/10.1016/03772217(91)90033-R

Xu, Z., y Liao, H. (2014). Intuitionistic fuzzy analytic hierarchy process. IEEE Transactions on Fuzzy Systems, 22(4), 749-761. doi:10.1109/TFUZZ.2013.2272585

Yang, C. C., y Chen, B. S. (2006). Supplier selection using combined analytical hierarchy process and grey relational analysis. Journal of Manufacturing Technology Management, 17(7), 926-941. doi:10.1108/17410380610688241 